\title{
Coordenação da atenção primária: limites e possibilidades para a integração do cuidado
}

\author{
Coordination of primary care: limits and possibilities for integration of care \\ Coordinación de la atención primaria: límites y posibilidades para la integración del cuidado
}

\author{
Tatiana Monteiro da Paixão'; Ana Inês Sousa"; \\ Maria Helena do Nascimento Souza ${ }^{\prime \prime \prime}$; Sheila Nascimento Pereira de Farias ${ }^{\text {IV }}$
}

\begin{abstract}
RESUMO
Objetivo: avaliar o atributo coordenação do cuidado na atenção primária à saúde na perspectiva de profissionais de equipes de saúde da família. Método: estudo seccional realizado com 349 profissionais de equipes saúde da família da área programática 3.1, por meio da aplicação do questionário Primary Care Assessment Tool. Estudo aprovado pelo Comitê de Ética em Pesquisa da Secretaria Municipal de Saúde do Rio de Janeiro. Resultados: o atributo coordenação do cuidado teve uma boa avaliação (escore 7,1), estando acima do ponto de corte esperado, 6,6. Observou-se um desconhecimento do fluxo dos usuários nos serviços especializados da rede de atenção à saúde (escore $=6,2)$ e uma deficiência no sistema de referência e contrareferência $($ escore $=4,6)$. Conclusão: evidenciou-se a necessidade da comunicação entre os profissionais da rede de atenção à saúde, a fim de melhorar o sistema de referência e contrareferência e possibilitar a integração dos cuidados prestados à população.

Descritores: Atenção primária à saúde; estratégia saúde da família; avaliação em saúde; enfermagem.
\end{abstract}

\section{ABSTRACT}

Objective: to evaluate the care coordination attribute of primary health care from the perspective of family health team personnel. Method: this cross-sectional study was carried out with 349 family health team professionals from program area 3.1, using the Primary Care Assessment Tool questionnaire. The study was approved by the Rio de Janeiro Municipal Health Secretariat research ethics committee. Results: the care coordination attribute was favorably assessed (scoring 7.1), above the expected cutoff point of 6.6. Knowledge of user flows in specialized services of the health care network was lacking (scoring 6.2), and the referral and counter-referral system was deficient (scoring 4.6). Conclusion: there was a need for communication among health care professionals in order to improve the referral and counter-referral system, and make it possible to integrate care provided to the population.

Descriptors: Primary health care; family health strategy; health evaluation; nursing.

\section{RESUMEN}

Objetivo: evaluar el atributo de coordinación del cuidado en la atención primaria de salud desde la perspectiva de los profesionales de equipos de salud familiar. Método: estudio seccional realizado junto a 349 profesionales de equipos de salud familiar del área del programa 3.1, a través de la aplicación del cuestionario Primary Care Assessment Tool. Estudio aprobado por el Comité de Ética en Investigación de la Secretaría Municipal de Salud de Río de Janeiro. Resultados: el atributo de coordinación del cuidado obtuvo una buena evaluación (puntaje 7.1), por encima del punto de corte esperado, 6.6. Se observó una falta de conocimiento sobre el flujo de usuarios en los servicios especializados de la red de atención a la salud (puntaje = 6.2) y una deficiencia en el sistema de referencia y contrarreferencia (puntaje $=4.6$ ). Conclusión: se puso en evidencia la necesidad de comunicación entre los profesionales de la red de atención a la salud, para mejorar el sistema de referencia y contrarreferencia y permitir la integración de la atención brindada a la población.

Descriptores: Atención primaria a la salud; estrategia de salud de la familia; evaluación en salud; enfermería.

\section{INTRODUÇÃO}

No modelo assistencial dos serviços de atenção primária, a coordenação do cuidado constitui em um atributo essencial e pressupõe a continuidade do atendimento pelo mesmo profissional ou mediante o encaminhamento para outros serviços, com vistas ao cuidado global do usuário ${ }^{1}$.

Estudos revelam fragilidades na coordenação do cuidado prestado pelos serviços de atenção primária, decorrentes da interrupção dos fluxos de atendimento, falta de comunicação entre os profissionais, uso deficiente dos recursos e do desequilíbrio entre a demanda e a oferta ${ }^{2,3}$. Além desses fatores, o fato das pessoas viverem em situações de grandes carências e vulnerabilidade, requer que os arranjos para a responsabilização e gestão do cuidado sejam muito mais complexos e articulados, pois uma coordenação do cuidado fraca constitui um importante obstáculo para a garantia da integralidade, do acesso e da oferta de serviços de saúde de qualidade ${ }^{4}$.

'Enfermeira. Mestranda. Escola de Enfermagem Anna Nery/ UFRJ. Rio de Janeiro, Brasil. E-mail: tatiana.monteiro.paixao@gmail.com

"Enfermeira. Doutora. Professora Titular da Escola de Enfermagem Anna Nery/ Universidade Federal do Rio de Janeiro. Brasil. E-mail: anainechico@gmail.com

I'Enfermeira. Doutora. Professora Associada da Escola de Enfermagem Anna Nery/ Universidade Federal do Rio de Janeiro. Brasil. E-mail: mhnsouza@yahoo.com.br

IVEnfermeira. Doutora. Professora Associada da Escola de Enfermagem Anna Nery/ Universidade Federal do Rio de Janeiro. Brasil. E-mail: sheilaguadagnini@gmail.com

VAgradecimento à agência de fomento Fundação de Amparo à Pesquisa do Estado do Rio de Janeiro pelo apoio financeiro à pesquisa, Edital FAPERJ N.o 28/2012. 
Diante do exposto, este estudo objetivou avaliar o atributo coordenação do cuidado na atenção primária à saúde na perspectiva de profissionais de equipes de saúde da família.

\section{REVISÃO DE LITERATURA}

A atenção primária à saúde, no contexto nacional e internacional, é considerada a porta de entrada dos sistemas de saúde. Através deste nível, os usuários são acompanhados no seu processo saúde-doença ou são referenciados para o nível secundário e/ou terciário quando necessitam. Desta forma, o acesso dos usuários à rede de atenção básica possibilita um cuidado integral, resolutivo e de qualidade ${ }^{5,6}$.

Segundo a Política Nacional de Atenção Básica, os cuidados primários em saúde são caracterizados por um conjunto de ações de saúde, no âmbito individual e coletivo, que abrange a promoção e proteção da saúde, a prevenção de agravos, o diagnóstico, o tratamento, a reabilitação, a redução de danos, e a manutenção da saúde com o objetivo de desenvolver uma atenção integral que impacte na situação de saúde e autonomia das pessoas e nos determinantes e condicionantes de saúde das coletividades ${ }^{5}$.

No Brasil, a principal estratégia de implementação da atenção primária à saúde tem sido a Estratégia de Saúde da Família (ESF). Esta estratégia vem a nível nacional como um modelo ordenador das redes de atenção à saúde, que se propõe a transformar o modelo tradicional assistencial biomédico, curativista, em um modelo de saúde centrado na atenção multiprofissional voltada para a promoção da saúde das famílias e comunidade ${ }^{5,7}$

Diante disso, há a necessidade de estudos voltados para a avaliação da qualidade e desempenho dos serviços oferecidos por meio da verificação dos atributos da APS. Nestas investigações, a visão dos profissionais poderá subsidiar a identificação de problemas ${ }^{8,9}$.

Os atributos essenciais englobam o primeiro contato, continuidade ou longitudinalidade do cuidado, integralidade e coordenação. Evidências científicas apontam que sistemas de saúde organizados a partir dos atributos ordenadores são eficazes e de qualidade ${ }^{9}$.

A coordenação ou integração de serviços envolve a facilidade em acessar os demais níveis de atenção e o acompanhamento do atendimento em outros serviços especializados, de forma hierarquizada, de acordo com as necessidades de saúde do usuário ${ }^{10}$.

\section{METODOLOGIA}

Trata-se de um estudo seccional realizado em Unidades de Saúde da Família situadas na área programática 3.1 do município do Rio de Janeiro, Brasil, extraído da pesquisa Serviços de Atenção Primária à Saúde do Município do Rio de Janeiro: uma análise das dimensões e potencialidades da Estratégia Saúde da Família, desenvolvida pelo Núcleo de Pesquisa de Enfermagem e Saúde Coletiva (NUPENSC) da Escola de Enfermagem Anna Nery da Universidade Federal do Rio de Janeiro.

A população do estudo foi constituída por 1284 profissionais de equipes de saúde da família: médicos, enfermeiros, dentista, técnicos de saúde bucal, auxiliares/técnicos de enfermagem e agentes comunitários de saúde (ACS), vinculados às unidades da Área de Planejamento selecionada.

Para a definição da amostra considerou-se o intervalo de confiança de $95 \%$, erro amostral de $5 \%$ e o percentual de $50 \%$ para o grau de homogeneidade da amostra.

Como critérios de inclusão foram considerados os profissionais que estavam prestando assistência nas equipes de saúde da família com período empregatício superior a quatro meses, a fim de possibilitar um maior contato temporal com a população atendida. Foram excluídos os profissionais que durante o período da coleta dos dados estavam de férias, licença médica ou licença maternidade e aqueles que não foram encontrados após três visitas das pesquisadoras à Unidade. Foram excluídos ainda os profissionais das unidades de saúde localizadas em uma região em que as pesquisadoras estiveram impossibilitadas de comparecer devido à falta de segurança.

Participaram do estudo 349 profissionais de saúde, sendo 86 enfermeiros, 24 médicos, 16 dentistas, 30 auxiliares e técnicos, 180 ACS, 13 técnicos de saúde bucal, selecionados por conveniência, na ocasião de visitas às unidades.

A coleta de dados ocorreu no período de janeiro a dezembro de 2014, por meio de entrevista com base no questionário de Avaliação da Atenção Primária Primary Care Assessment Tool (PCATool) - versão para Profissionais, validado para uso no Brasil. ${ }^{11}$ Deste questionário foram selecionadas as questões referentes ao atributo da coordenação - integração do cuidado. Além destas questões foram acrescentadas no instrumento variáveis sociodemográficas e ocupacionais para possibilitar a caracterização dos participantes.

Os dados foram processados e analisados com auxílio dos programas Epi-Info versão 3.5 e Statistical Package for the Social Sciences (SPSS) versão 21. 
O cálculo dos escores, dispostos em uma escala do tipo Likert, foi realizado de acordo com os passos descritos no manual do instrumento PCATool ${ }^{11}$. As respostas possíveis para cada um dos itens são: "com certeza sim" valor=4, "provavelmente sim" valor=3, "provavelmente não" valor=2, "com certeza não" valor=1 e "não sei/ não lembro" valor=9. Assim, inicialmente foi realizada a inversão dos valores dos escores, quando indicada. Para cada componente calculou-se um escore médio, baseado no quociente entre a soma do valor dos itens e o número de itens.

Os escores foram convertidos em uma escala de 0 a 10 mediante a seguinte fórmula: [escore obtido -1 valor mínimo] X 10 / 4 valor máximo - 1 valor mínimo. Os valores iguais ou superiores a 6,6 indicaram satisfação para com o determinado atributo da atenção primária ${ }^{11}$.

Foram respeitados os aspectos éticos e legais exigidos pela Resolução 466/2012 e o estudo foi aprovado pelo Comitê de Ética em Pesquisa da Secretaria Municipal de Saúde do Rio de Janeiro sob o protocolo no. 90/13, e autorizado pela Coordenação de Área Programática 3.1 do município do Rio de Janeiro.

\section{RESULTADOS}

Com relação às características da amostra estudada, verificou-se que a maioria dos profissionais entrevistados apresentou idade inferior a 34 anos e era do sexo feminino. Em relação à escolaridade verificou-se o predomínio de profissionais com Ensino Médio Completo, o que pode ser explicado pela grande quantidade de Agentes Comunitários de Saúde e de Técnicos entrevistados. Quanto ao tempo de atuação na Saúde da Família e na Unidade de saúde atual, a média foi de 3 anos e 2,5 anos, respectivamente (Tabela 1).

TABELA 1: Características sociodemográfico e ocupacionais dos profissionais entrevistados. Rio de Janeiro, RJ, Brasil, 2014.

\begin{tabular}{|c|c|c|c|c|c|c|}
\hline \multirow{2}{*}{\multicolumn{7}{|c|}{$f(\%)$}} \\
\hline & & & & & & \\
\hline$\leq 34$ & $84(47,2)$ & $9(60,0)$ & $52(60,5)$ & $18(75,0)$ & $13(44,8)$ & $5(38,5)$ \\
\hline$>34$ & $94(52,8)$ & $6(40,0)$ & $34(39,5)$ & $6(25,0)$ & $16(55,2)$ & $8(61,5)$ \\
\hline \multicolumn{7}{|l|}{ Sexo } \\
\hline Feminino & $150(83,3)$ & $14(87,5)$ & $77(89,7)$ & $15(62,5)$ & $26(86,2)$ & $13(100)$ \\
\hline Masculino & $30(16,7)$ & $2(12,5)$ & $9(10,3)$ & $9(37,5)$ & $4(13,8)$ & $0(0,0)$ \\
\hline \multicolumn{7}{|l|}{ Escolaridade } \\
\hline Até Ensino Médio & $165(91,7)$ & - & - & - & $29(96,7)$ & $12(92,3)$ \\
\hline Ensino Superior & $13(7,2)$ & $2(12,5)$ & $23(26,7)$ & $11(45,8)$ & $1(3,3)$ & $1(7,7)$ \\
\hline Pós-graduação. & $2(1,1)$ & $14(87,5)$ & $63(73,3)$ & $13(54,2)$ & - & - \\
\hline \multicolumn{7}{|c|}{$\begin{array}{l}\text { Tempo de atuação em } \\
\text { Saúde da Família (em anos) }\end{array}$} \\
\hline$\leq 3$ & $125(69,9)$ & $6(37,5)$ & $38(44,2)$ & $17(70,8)$ & $17(56,7)$ & $3(23,1)$ \\
\hline$>3$ & $55(30,6)$ & $10(62,5)$ & $48(55,8)$ & $7(29,2)$ & $13(43,3)$ & $9(69,2)$ \\
\hline \multicolumn{7}{|c|}{$\begin{array}{l}\text { Tempo de atuação na } \\
\text { unidade de Saúde da Família } \\
\text { atual (em anos) }\end{array}$} \\
\hline$\leq 2,5$ & $68(39,5)$ & $4(28,5)$ & $52(63,4)$ & $18(85,7)$ & $14(48,3)$ & $5(38,5)$ \\
\hline$>2,5$ & $104(60,5)$ & $10(71,5)$ & $30(36,6)$ & $3(14,3)$ & $15(51,7)$ & $8(61,5)$ \\
\hline
\end{tabular}

No que tange ao escore geral do atributo coordenação - integração dos cuidados foi calculado um escore de 7,1 com desvio padrão de 1,66. A Tabela 2 apresenta a distribuição dos escores de acordo com as questões que avaliam esse atributo.

Pode-se observar que, em geral, o atributo Coordenação - Integração de cuidados foi bem avaliado, tendo um escore de 7,1, estando acima do escore de corte 6,6 estabelecido pelo manual do instrumento. No entanto, os profissionais não demonstraram satisfação com relação ao conhecimento do tipo consulta que seus pacientes fazem com especialistas e a falta de informação dos serviços para onde os pacientes foram encaminhados. 
TABELA 2: Distribuição das respostas referentes ao atributo coordenação - integração dos cuidados. Rio de Janeiro, RJ, Brasil, 2014.

\begin{tabular}{lc}
\hline Questões & Escore médio \\
\hline $\begin{array}{l}\text { Você tem conhecimento de todas as consultas que seus pacientes fazem a especialistas ou } \\
\text { serviços especializados? }\end{array}$ & 6,2 \\
$\begin{array}{l}\text { Quando seus pacientes necessitam de um encaminhamento, você discute com os pacientes } \\
\text { sobre os diferentes serviços onde eles poderiam ser atendidos? }\end{array}$ & 7,0 \\
$\begin{array}{l}\text { Alguém de seu serviço de saúde ajuda o paciente a marcar a consulta encaminhada? } \\
\text { Quando seus pacientes são encaminhados, você lhes fornece informação escrita para levar }\end{array}$ & 8,9 \\
ao especialista ou serviço especializado? & 8,8 \\
$\begin{array}{l}\text { Você recebe do especialista ou do serviço especializado informações úteis sobre o paciente } \\
\text { encaminhado? }\end{array}$ & 4,6 \\
$\begin{array}{l}\text { Após a consulta com o especialista ou serviço especializado, você fala com seu paciente } \\
\text { sobre os resultados desta consulta? }\end{array}$ & 7,3 \\
\hline
\end{tabular}

Dentre os profissionais, os dentistas demonstraram maior satisfação com as ações referentes à coordenação dos cuidados na atenção primária à saúde, enquanto que os técnicos de enfermagem referiram insatisfação (Tabela 3).

TABELA 3: Distribuição dos escores médios de acordo com a categoria profissional. Rio de Janeiro, RJ, Brasil, 2014

\begin{tabular}{lccc}
\hline Categoria Profissional & Escore médio & Desvio padrão & P \\
\hline Enfermeiro & 7,36 & 1,33 & \\
Médico & 7,43 & 1,20 & \\
Dentista & 8,22 & 1,74 & $<0,001$ \\
Técnico de enfermagem & 5,74 & 2,06 & \\
Técnico de saúde bucal & 8,11 & 1,90 & \\
Agente Comunitário de saúde & 7,13 & 1,61 & \\
\hline
\end{tabular}

\section{DISCUSSÃO}

Os resultados deste estudo corroboram com os de pesquisa similar no âmbito da atenção primária à saúde, que evidenciam a presença de profissionais jovens, com idade entre 20 e 29 anos, maioria do sexo feminino, com tempo de atuação na unidade de saúde da família entre um a 5 anos ${ }^{12}$.

É função da atenção primária coordenar as ações de forma compartilhada entre os diversos atores da rede de atenção à saúde, garantindo a continuidade do cuidado. No entanto, esta coordenação nem sempre acontece de modo eficiente e eficaz. E, quando os serviços não se comunicam, o cuidado ao usuário se torna fragmentado. ${ }^{13}$ Nesta perspectiva, diversos estudos apontam para a relevância de investigações sobre as formas de organização e gestão dos serviços da rede de atenção primária à saúde ${ }^{5,7,8,12,14}$.

Os resultados mostraram um desconhecimento do fluxo dos usuários nos serviços especializados da rede de atenção à saúde (escore=6,2) e uma deficiência no sistema de referência e contrareferência (escore=4,6). Outros estudos realizados com objetivo de avaliar a coordenação da atenção primária revelaram que este atributo foi bem avaliado pelos profissionais, entretanto os pesquisadores ressaltam a avaliação negativa na questão referente ao funcionamento da contrarreferência ${ }^{12,14}$.

A referência e contrareferência são métodos considerados eficazes de seguimento do fluxo do usuário no sistema de saúde e também de vinculação. Quando esses métodos não funcionam geram problemas que afetam o cuidado prestado ao usuário, pois este fica sem um direcionamento no sistema de saúde, o que impossibilita o acompanhamento de sua condição de saúde de forma integral ${ }^{15,16}$.

Outra implicação da não realização da contrareferência está centrada no aumento de gastos para a área de saúde, pois ocorre a duplicidade de trabalho ${ }^{15,17}$. Além disso, o déficit de recursos humanos é outro fator que pode ser prejudicial para a realização da articulação entre os profissionais dos três níveis de atenção à saúde e consequentemente interferir na qualidade da assistência ${ }^{4,13}, 18$. 
No que concerne à lógica da articulação dos serviços, esta deve ser percebida inclusive no interior do mesmo nível de atenção, entre as diversas categorias profissionais ${ }^{19}$, diminuindo a lacuna existente na integração dos cuidados prestados aos usuários por todos os integrantes da equipe de saúde. Neste sentido, através da atuação das equipes de saúde da família, evidencia-se uma maior capilaridade do sistema de saúde, o que permite maior planejamento, comunicação e coordenação do cuidado no âmbito dos níveis primário, secundário e terciário da rede de atenção à saúde ${ }^{20-22}$

Entre as limitações do estudo destaca-se o fato dos resultados obtidos expressarem a realidade específica de profissionais que atuam em unidades de saúde da família de uma das dez áreas de planejamento do município do Rio de Janeiro. Além disso, a situação de violência urbana e a sobrecarga de trabalho impossibilitaram que a coleta de dados fosse realizada junto à totalidade dos profissionais identificados. O tipo de delineamento desta pesquisa sendo transversal também pode ser considerado uma limitação, uma vez que não permitiu algumas correlações e inferências causais na análise dos escores atribuídos pelos profissionais à qualidade dos serviços prestados na unidade de saúde da família.

Desta forma, destaca-se a necessidade do desenvolvimento de outras pesquisas mais abrangentes que permitam a generalização dos resultados e o preenchimento da lacuna existente na produção do conhecimento sobre a temática da avaliação da atenção primária à saúde no Brasil.

$\mathrm{O}$ artigo aponta para a necessidade de refletir e estabelecer uma melhor e mais efetiva comunicação entre os profissionais generalistas e especialistas, como forma de garantir um cuidado integral e ampliar a oferta aos serviços de saúde.

Além disso, destaca a necessidade de guiar o usuário dentro do sistema de saúde por meio de referência e contrareferência qualificada, estabelecendo vínculo e a continuidade da assistência.

Sendo assim, contribui para a prática profissional e acadêmica, podendo ser utilizado como estudo piloto para novas pesquisas.

\section{CONCLUSÃO}

Acredita-se que o presente estudo forneceu subsídios para a reflexão acerca da qualidade da coordenação do cuidado voltado para a população na atenção primária à saúde, a partir do conhecimento dos limites e possibilidades apontados pelos profissionais de equipes de saúde da família.

Apesar do escore médio do atributo avaliado estar acima da média, este estudo evidencia a necessidade da comunicação entre os profissionais da rede de atenção à saúde, a fim de melhorar o sistema de referência e contrareferência e possibilitar a integração dos cuidados prestados à população.

Ressalta-se, ainda, que a avaliação periódica dos serviços de atenção primária é uma importante ferramenta para a implementação de ações intersetoriais e para a garantia da continuidade do cuidado integral, acesso equitativo e fluxo dos usuários na rede de atenção à saúde.

\section{REFERÊNCIAS}

1. Starfield B. Primary Care: balancing health needs, services, and technology. Brasília: Organização das Nações Unidas para a Educação, a Ciência e a Cultura, Ministério da Saúde; 2002. [cited 2018 Jan 07]. Available from:

http://bvsms.saude.gov.br/bvs/publicacoes/atencao_primaria_p1.pdf

2. Cecilio LCO, Andreazza RCG, Araújo EC, Oliveira LA, Andrade MGG, Meneses CS et al. Primary Health Care and the construction of thematic health networks: what can be its role? Ciênc. saúde coletiva [Internet]. 2012 [cited 2018 Jan 07]; 17(11): $2893-2902$. DOI: http://dx.doi.org/10.1590/S1413-81232012001100006

3. Mendes EV. Access to Primary Health Care. Brasília. Conselho Nacional de Secretários de Saúde - CONASS; 2017.193 p.: il.

4. Bousquat A, Giovanella L, Campos EMS, Almeida PF, Martins CL, Mota PHS et al. Primary health care and the coordination of care in health regions: managers' and users' perspective. Ciênc. saúde coletiva [Internet]. 2017 [cited 2018 Nov 21]; 22(4): 1141-1154. DOI: http://dx.doi.org/10.1590/1413-81232017224.28632016

5. Ministério da Saúde (BR). Departamento de Atenção Básica, Secretaria de Atenção à Saúde. Política Nacional de Atenção Básica. Brasília: Ministério da Saúde; 2017.

6. Hansen J, Groenewegen PP, Boerma WG, Kringos DS. Living in country with a strong primary care system is beneficial to people with chronic conditions. Health affairs. [Internet] 2015 [cited 2018 Nov 20]; 34(9):1531-1537. Available from: https://www.healthaffairs.org/doi/abs/10.1377/hlthaff.2015.0582

7. Silva SA, Baitelo TC, Fracolli LA. Primary Health Care Evaluation: the view of clients and professionals about the Family Health Strategy. Rev. Latino-Am. Enfermagem. [Internet]. 2015 [cited 2018 Jan 07]; 23(5): 979-987. DOI: http://dx.doi.org/10.1590/0104-1169.0489.2639

8. Barber RM, Fullman N, Sorensen RJD, Bollyky T, McKee M, Nolte E et al. Healthcare access and quality index based on mortality from causes amenable to personal health care in 195 countries and territories, 1990-2015: a novel analysis from the global 
burden of disease study 2015. Lancet. [Internet] 2017 [cited 2018 Nov 20]; 390(10091):231-66. DOI: http://dx.doi.org/10.1016/S0140-6736(17)30818-8

9. Vieira NF, Lanza FM, Lana FCF, Martínez-Riera JR. Assessment of the attributes of primary health care in leprosy control actions. Rev. enferm. UERJ. [Internet] 2018 [cited 2019 Sep 23]; 26:e31925. DOI: https://doi.org/10.12957/reuerj.2018.31925

10. Marin MJS, Moracick MYAD, Marchioli M. Health service access: comparing professionals' and users' views of primary care. Rev. enferm. UERJ. [Internet] 2014 [cited 2019 May 23]; 22(5):629-36. Available from: https://www.epublicacoes.uerj.br/index.php/enfermagemuerj/article/view/4238/12282

11. Ministério da Saúde (BR). Secretaria de Atenção em Saúde. Departamento de Atenção Básica. Primary care assessment tool pcatool. Brasília: Ministério da Saúde; 2010. [cited 2018 Jun 14]. Available from: http://bvsms.saude.gov.br/bvs/publicacoes/manual_avaliacao_atencao_primaria.pdf

12. Silva SA, Nogueira DA, Paraizo CMS, Fracolli LA. Assessment of primary health care: health professionals' perspective. Rev. esc. enferm. USP [Internet]. 2014 [cited 2018 Jun 14]; 48(spe):122-128. DOI: http://dx.doi.org/10.1590/S0080623420140000600018

13. Protasio APL, Silva PB, Lima EC, Gomes LB, Machado LS, Valença AMG. Evaluation of the reference and counter-reference system based on the responses of the Primary Care professionals in the first External Evaluation cycle of PMAQ-AB in the state of Paraíba. Saúde debate [Internet]. 2014 [cited 2018 Jan 07]; 38(spe):209-220. DOI: http://dx.doi.org/10.5935/01031104.2014S016

14. Mazutti-Penso J, Périco E, Oliveira M, Guimarães-Strohschoen A, Carreno I, Rempel C. Evaluation of primary health care using the PCATool-Brazil instrument. Rev. bras. med. fam. Comunidade. [Internet]. 2017 [cited 2018 Nov 20]; 12(39):1-9. Available from: https://www.rbmfc.org.br/rbmfc/article/view/1212

15. Morato MGC, da Silva Júnior JLR, Carneiro DA, Sacchetim SC, Taleb AC, Rabahi MF. Strategy analysis for application of electronic system in reference and counter-reference in health services. Rev. Educ. Saúde [Internet] 2015; 3(1):20-30 [cited 2018 Jun 14]. Available from: http://revistas.unievangelica.edu.br/index.php/educacaoemsaude/article/view/1239/1147

16. Alves MLF, Guedes HM, Martins JCA, Chianca TCM. Reference and counter reference network for emergency care assistance in a municipality in the countryside of Minas Gerais - Brazil. Rev. Med. Minas Gerais [Internet]. 2015; 25(4):469-75. [cited 2018 Jun 14]. DOI: http://dx.doi.org/10.5935/2238-3182.20150110

17. Chomatas ERV, Vigo A, Marty IK, Hauser L, Harzheim E. Evaluation of the presence and extension of attributes of primary care in Curitiba. Rev. bras. med. fam. Comunidade. [Internet]. 2013 [cited 2018 Jun 14]; 8(29):294-303. DOI: https://doi.org/10.5712/rbmfc8(29)828

18. Costa PHA, Martins LF, Medeiros AX, Salgado JA, Silva WMD, Ronzani TM et al. Referral and counter-referral system in the drug user care network: contributions from social network analysis. Cad. saúde colet. [Internet]. 2015 [cited 2018 Jan 07]; 23(3): 245252. DOI: http://dx.doi.org/10.1590/1414-462X201500030129

19. Almeida PF, Santos AM. Primary Health Care: care coordinator in regionalized networks? Rev. Saúde Pública [Internet]. 2016 [cited 2018 Nov 21]; 50:80. DOI: http://dx.doi.org/10.1590/s1518-8787.2016050006602

20. Batista VCL, Ribeiro LCC, Ribeiro CDAL, Paula FA, Araújo A. Evaluation of the attributes of primary health care according to family health professionals. Sanare (Sobral, Online). 2016 [cited 2018 Nov 21]; 15(02):87-93. Available from: https://sanare.emnuvens.com.br/sanare/article/view/1042

21. Papp R, Borbas I, Dobos E, Bredehorst M, Jaruseviciene L, Vehko T et al. Perceptions of quality in primary health care: perspectives of patients and professionals based on focus group discussions. BMC Fam Prac. [Internet] 2014; 15:128. [cited 2018 Dec 10]. DOI: http://dx.doi.org/10.1186/1471-2296-15-128

22. Brondani JE, Leal FZ, Potter C, Silva RM, Noal HC, Perrando MS. Challenges of reference and counterfeiting in health care in workers perspective. Cogitare enferm. Rev. [Internet]. 2016 [cited 2018 Dec 10]; 21(1):2176-9133. DOI: http://dx.doi.org/10.5380/ce.v21i1.43350 